\title{
Gene Expression and Microarray Investigation of Dendrobium Mixture as Progressive Therapy for the Treatment of Type 2 Diabetes Mellitus
}

\author{
Qian Xu' ${ }^{1}$, Yun Liu ${ }^{1}$, Yi-Bo Cong ${ }^{2}$, Yuan-Yan Zheng ${ }^{1}$, Jie-Ping Zhang ${ }^{1}$, Yi Cui ${ }^{3}$ \\ and Hong Shi ${ }^{1 *}$ \\ ${ }^{1}$ Institute of Integrated Chinese and Western Medicine, Fujian University of Traditional Chinese Medicine; \\ ${ }^{2}$ College of Nursing, Fujian University of Traditional Chinese Medicine; ${ }^{3}$ Biomedical Institute, Fujian Medical \\ University, Fuzhou, China.
}

*For correspondence: E-mail: shihong3327@sina.com; Tel: +86-591-22861151; Fax: +86-591-22861151;

\begin{abstract}
Purpose: To examine the gene expression profile, as well as blood sugar-lowering and lipid-lowering molecular mechanisms of Dendrobium mixtures in a diabetic rat model

Methods: Sprague Dawley (SD) rats were fed high-fat/high-glucose for 16 months. Those with random blood glucose $>16.7 \mathrm{mmol} / \mathrm{L}$ were used as the model group and treated with Dendrobium mixture (DEN, containing Dendrobium, Astragalus, Schisandra, etc) in clinically equivalent dose (12 g/kg). The liver RNA of the rats in all three groups (control, model and DEN) was used for Agilent genome expression microarray testing and subsequent data analysis.

Result: Between the diabetic rat group and the wild-type group, 1339 functional genes showed differences in expression levels ( $p<0.05)$. After Dendrobium treatment, only 380 genes showed differences in expression ( $p<0.05)$. The expression level of nearly 1000 genes returned to normal after drug treatment (compared with the wild-type group, $p>0.05$ ). Genes whose expression normalized were mainly those affected by the disease state and associated with glucose and lipid metabolism, cell growth, apoptosis, biosynthesis, olfactory receptors, or cytoskeletal proteins.

Conclusion: Progressive therapy with Dendrobium mixture, which has glucose- and lipid-lowering effects, is associated with multi-gene expression pathways. By treating diabetic $r$ and wild-type rats with the mixture, the disorder is further understood at the transcriptomic level.
\end{abstract}

Keywords: Diabetes, Gene expression, Dendrobium mixture, Microarray testing

Tropical Journal of Pharmaceutical Research is indexed by Science Citation Index (SciSearch), Scopus, International Pharmaceutical Abstract, Chemical Abstracts, Embase, Index Copernicus, EBSCO, African Index Medicus, JournalSeek, Journal Citation Reports/Science Edition, Directory of Open Access Journals (DOAJ), African Journal Online, Bioline International, Open-J-Gate and Pharmacy Abstracts

\section{INTRODUCTION}

With social and economic development, improvement in quality of life, and increasing life expectancy, diabetes mellitus (DM) has shown increasing prevalence, becoming a global health issue. Type 1 diabetes is a chronic autoimmune disease triggered by environmental factors, with islet B cell-specific injury; type 2 diabetes is due to insufficient insulin secretion or peripheral tissue insulin resistance, accounting for more than $95 \%$ of cases in adult patients[1-2]. The pathogenesis of diabetes is unclear; although cellular and animal experiments, and clinical tests have shown that hypoglycaemic symptomatic treatment has a protective effect[3]. 
Over the past decade, many studies have reported the use of traditional Chinese medicine (TCM) for the treatment of diabetes mellitus in clinical and experimental research. Gene chip, also called cDNA microarray, refers to the many specific gene fragments fixed to a solid support. The tagged samples are hybridized with the fixed fragment on the solid support. By confocal laser scanning fluorescence detection system, hybridization signal intensity is detected and the number of molecules and sequence information are obtained. Using computer software for data comparison and analysis, gene sequence and function are studied on a large-scale and highthroughput [4]. Its purpose is to extract information from genes with an appropriate clustering method [5].

This study presents a theoretical basis for therapy of diabetes by searching for potential drug targets in the genome.

\section{EXPERIMENTAL}

\section{Experimental animals}

Seventy healthy female specific pathogen-free (SPF) SD rats, 12 weeks old, weighing $200 \pm 20$ $\mathrm{g}$, were provided by Slac Laboratory Animal (Shanghai, China). All animal experiments were performed in accordance with the approval received from the Institutional Animal Ethics Committee following the guidelines of the Committee for the Purpose of Control and Supervision of Experiments on Animals (CPCSEA), Government of China, and the International Guidelines for Handling of Laboratory Animals [6].

\section{Plant material}

Dendrobium (Dendrobium officinale Kimura et Migo), belongs to the orchid species, and mainly distributed in tropical and subtropical Asia, Australia and the Pacific Islands, and the southern region of China. The plant material was collected from the wild in Fujian province in south China in April 2010 and authenticated by Associate Professor Chengzi Yang at the Department of Pharmacy, Fujian Medical University, Fuzhou, China; a voucher specimen (XQ-2010) was deposited in the herbarium of Integrative Medicine, Fujian University of Traditional Chinese Medicine, Fuzhou, China. The plant was dried to preserve it.

\section{Preparation of Dendrobium mixtures}

Dendrobium mixture 1 consisted of Dendrobium $15 \mathrm{~g}$, astragalus $15 \mathrm{~g}$, Schisandra $20 \mathrm{~g}$, salvia 20 $\mathrm{g}$, arrowroot $15 \mathrm{~g}$, yam $10 \mathrm{~g}$ and dried rehmannia root $10 \mathrm{~g}$; while Dendrobium mixture 2 consisted of Dendrobium $15 \mathrm{~g}$, astragalus $20 \mathrm{~g}$, Schisandra $10 \mathrm{~g}$, salvia $10 \mathrm{~g}$, arrowroot $15 \mathrm{~g}$, dried rehmannia root $10 \mathrm{~g}$, Scrophulariaceae 10 $\mathrm{g}$, Anemarrhena $10 \mathrm{~g}$, habitat $10 \mathrm{~g}$, gypsum, 20 $\mathrm{g}$, Cyathula $10 \mathrm{~g}$, berberine $10 \mathrm{~g}$. Dendrobium mixture 3 was composed of Capillaris $20 \mathrm{~g}$, rhubarb $4 \mathrm{~g}$, gardenia $10 \mathrm{~g}$ and Bupleurum $6 \mathrm{~g}$. After mixing the ingredients of the formulation, it was soaked in $500 \mathrm{ml}$ of water for $1 \mathrm{~h}$ and then boiled for one hour to brew. It was sterilized for $20 \mathrm{~min}$ at $200{ }^{\circ} \mathrm{C}, 100 \mathrm{ml}$ of the mixture was packed into separate glass bottles and kepted for gavage experiments.

\section{Pretreatment of animals}

The SD rats (12 weeks old) were adaptively fed for 7 days in the Fujian University of Traditional Chinese Medicine SPF level Animal Center Laboratory, and then randomly divided into two groups: 15 rats in the wild-type group and 55 rats in the high-fat/high-glucose group. The wild-type group was given a basic diet while the highfat/high-glucose group was given a high-fat/highglucose diet. The basic diet, obtained from Fujian Medical University Experimental Animal Center, composed of $19 \%$ flour, $23 \%$ corn flour, 6 \%sorghum flour, $10 \%$ wheat bran, $20 \%$ skim milk powder, $10 \%$ fish meal, $7 \%$ starch, $3 \%$ glycine and $2 \%$ of the beer yeast. The highfat/high-glucose diet was prepared to contain 15 $\%$ sugar, $10 \%$ lard, $4 \%$ cholesterol, $0.3 \%$ bile salts, $10 \%$ egg yolk powder, and $60.7 \%$ basic diet. The rats were continuously fed for 16 months, and blood glucose monitored by a glucose meter. One wild-type rat and 4 highfat/high-glucose rats died during the test. If model generation failed (note: model generation succeeded if random blood glucose was $\geq 16.7$ $\mathrm{mmol} / \mathrm{L}$, and unsuccessful if $<16.7 \mathrm{mmol} / \mathrm{L}$ ), then intraperitoneal injection of a low dose of streptozotocin (STZ) was given (20 mg/kg), under ice bath conditions, $0.25 \%$ sodium citrate buffer ( $\mathrm{pH} 4.2$ ), prepared immediately prior to use). Successfully generated diabetic rats were selected 3 days later using a small-volume glucometer for blood sugar measurement. Low doses of STZ were injected into the remaining non-diabetic rats. Successfully generated model rats (random blood glucose $\geq 16 \mathrm{mmol} / \mathrm{L}$ ) were again selected 3 days later and all diabetic rats were randomly divided into the diabetic rat model group and Dendrobium mixture group.

\section{Treatment of animals}

Initially, the Dendrobium mixture (DEN) was gavag to the DEN treatment group at a dos of 12 
$\mathrm{g} /(\mathrm{kg} / \mathrm{d})$ (clinically equivalent dose): Dendrobium mixtures (DEN) 1 and 2 were administered for 6 days and 12 days to the DEN treatment group, respectively. Thereafter, the rats were fed DEN 3 for 3 days as a course of treatment. The animals were allowed a wahout for a day in-between the treatment types. Two courses of treatment (DEN

1, 2 and) were conducted over a total period of 48 days.

After two dosage cycles (DEN 1, 2 and 3 twice each), the rats were fasted overnight and anesthetized using $1 \%$ urethane after first weighing them. Rapid laparotomy was then performed, liver tissue was removed and stored in liquid nitrogen. Tissue samples were removed in this way from randomly selected rats in each of the wild-type, diabetic model, and treatment groups and sent to Shanghai Biotechnology Co, Ltd for Agilent Whole Rat Genome Oligo Microarray $(4 \times 44 K)$ testing.

\section{Total RNA extraction and quality control}

Total RNA was extracted from $1 \mathrm{~g}$ of liver tissue using $2 \mathrm{ml}$ Trizol reagent following the standard protocols provided by the manufacturer of the reagent. After passing the Agilent Bioanalyzer 2100 electrophoresis quality assessment, RNeasy mini-kit and RNase-free DNase kit were used to purify the total RNA. If RNA quality control result for 2100 RIN and 28 S/18S was $\geq$ 7.0 and $\geq 0.7$, respectively, then the test sample qualified for further experiments.

\section{Microarray test}

Sample RNA was treated with an Agilent microarray matching kit and a single-color Low Input Quick Amp Labeling Kit. The mRNA of the total RNA sample was amplified and labeled using standard protocols, and then labeled cRNA purified with an RNeasy mini kit. According to the standard protocol provided by the Agilent Gene Expression Hybridization Kit, hybridization reactions were rotated for $17 \mathrm{~h}$ in a rotating hybridization oven at $65{ }^{\circ} \mathrm{C}$ and $10 \mathrm{rpm}$. A sample $(1.65 \mu \mathrm{g})$ of hybridized cRNA was obtained and washed. The the hybridized chip was scanned in an Agilent Microarray Scanner and the Gene Spring Software 11.0 (Agilent Technologies, Santa Clara, CA, U.S.) was applied for normalization using Quantile algorithm.

\section{Data analysis}

The online SAS analysis system (at Shanghai Biotechnology Co, Ltd) was used for bioinformatics analysis. The R-package within the software package was used for statistical analysis. The samples from the three groups were compared using single-factor analysis of variance (one-way ANOVA). Pairwise comparisons between groups were performed with multiple comparisons Dunnett-t test. Significant differences were filtered by setting the $p$-value at $<0.05$, and the FC (fold change, also called difference factor) value calculated. A given gene was categorized as upregulated when $\mathrm{FC} \geq$ 2 and as downregulated when $\mathrm{FC} \leq 0.5$. The result file was used for database comparison and comments. The basic annotation and information for various genes were taken from the NCBI Entrez Gene database (http://www.ncbi.nlm .nih.gov/gene/) while the gene pathway data were taken from KEGG (http://www.genome. jp/kegg/) and Biocarta (http://www.biocarta.com/) databases.

\section{RESULTS}

The differences in expression of all genes in the two groups are displayed on a scatter plot (Fig 1). The $X$ and $Y$ axes are coordinates of the fluorescence signal intensity value of the two samples, each data point represents a gene hybridization signal on the chip. Signals that showed differential expression fell outside of the parallel lines, where fold change was $>2$. The left-side data points outside of the parallel lines in the left figure reflect increased gene expression in the model and wild-type groups while data points to the right reflect downregulation. The data points on the left side of the parallel lines indicate increased expression of genes between the model and treatment groups, while data points to the right side show down-regulated expression of the genes.

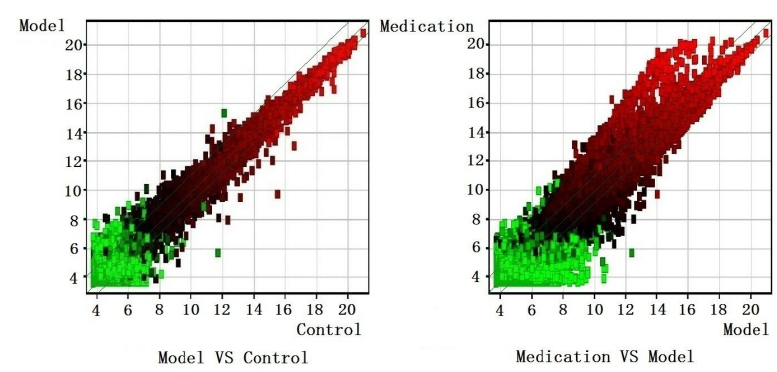

Fig 1: Scatterplot constructed with GeneSpring 11.0. Note: Model = rat model group; medication = treatment group, and control = wild-type control group

As a result, 1678 differentially expressed genes $(p<0.05)$ were identified between the wild-type and diabetic model groups. Of these, the functions of 1339 are known while those of 399 are unknown. Of the genes originally displaying FC > 2, 78 were downregulated and 182 upregulated. There were 1107 differentially 
expressed genes $(p<0.05)$ identified between the treatment and the model groups. Of these, the functions of 671 are known and those of 336 are unknown. Among the genes originally displaying $\mathrm{FC}>2,32$ were subsequently downregulated and 277 upregulated. There were 665 differentially expressed genes identified between the treatment and the wild-type groups $(p<0.05)$. Of these, 380 are known genes and 285 are unknown. Among genes subjected to pre-treatment $\mathrm{FC}>2,20$ were found to have been downregulated and 59 upregulated.

Overall, pairwise comparison for the three groups indicates that post-treatment diabetic rats expressed about 1,000 genes near wild-type levels, but the difference was not statistically significant $(p>0.05)$. The expression levels of these genes were considered to have been corrected. A few genes displayed post-treatment directionality of regulation similar to that observed in the diabetic group, but their differences in expression level relative to the wild-type group were equal to or less than their differences in expression level from the diabetic group. In addition, no genes displaying growth bias were found. Tables 1 and 2 show the correction of the down- and up-regulated genes in the treatment group $(p<0.05)$. Fig.2 shows the chromosomal localization of genes by ANOVA.

Table 1: Down-regulation in diabetic rat model indicating normally regulated genes after dendrobium mixture treatment

\begin{tabular}{lcccc}
\hline Gene & Abbreviation & Gene Id & Chromosome & FC \\
\hline SH3 and cysteine rich domain 3 & Stac3 & 362895 & 7 & 0.0203 \\
Stearoyl-CoA desaturase (delta-9-desaturase) & Scd & 83792 & 1 & 0.0739 \\
Major urinary protein 5 & Mup5 & 298107 & 5 & 0.1126 \\
Alpha-2u globulin PGCL3 & LOC259244 & 259244 & 5 & 0.1426 \\
Patatin-like phospholipase domain containing 5 & Pnpla5 & 300108 & 7 & 0.1755 \\
Similar to major urinary protein 5 & LOC685577 & 685577 & 5 & 0.1926 \\
Torsin family 3, member A & Tor3a & 304884 & 13 & 0.2265 \\
Similar to RIKEN cDNA B130016010 gene & RGD1308448 & 310358 & 2 & 0.2497 \\
\hline
\end{tabular}

Note: Gene Id represents the identity (ID) of the gene in GenBank; FC = model group gene expression level/wild-type group expression level; genes with FC $<0.25$ are listed in this table. With respect to chromosomal localization, "-" indicates multiple chromosomal locations for a single gene; "Un" indicates unknown

Table 2: Up-regulation in diabetic rat model after administration of Dendrobium mixture indicating genes that show normal expression

\begin{tabular}{lllcc}
\hline Gene & Abbreviation & Gene Id & Chromosom & FC \\
\hline Glyoxalase domain containing 5 & Glod5 & 302554 & X & 17.3097 \\
Regulator of G-protein signaling 4 & Rgs4 & 29480 & 13 & 11.8168 \\
Protein phosphatase 2, regulatory subunit B, beta isoform & Ppp2r2b & 60660 & 18 & 8.7967 \\
Similar to TGF beta-inducible nuclear protein 1 (LNR42) & LOC679999 & 679999 & X & 8.1652 \\
Heat shock protein 1 & Hspb1 & 24471 & 12 & 7.8923 \\
Ring finger protein 186 & Rnf186 & 690433 & 5 & 7.4041 \\
Glutathione peroxidase 2 & Gpx2 & 29326 & 6 & 5.4068 \\
Follistatin & Fst & 24373 & 2 & 5.1331 \\
Brain expressed gene 1 & Bex1 & 501625 & X & 4.5534 \\
Gamma-glutamyltransferase 1 & Ggt1 & 116568 & - & 4.55 \\
Integrin, beta 6 & Itgb6 & 311061 & 3 & 4.3516 \\
WNT1 inducible signaling pathway protein 2 & Wisp2 & 29576 & 3 & 4.2208 \\
Glypican 3 & Gpc3 & 25236 & X & 4.1231
\end{tabular}

Note: Gene Id represents the ID of the gene in GenBank; FC = model group gene expression level/wild-type group expression level; genes with FC > 4 are listed in this table; with respect to chromosomal localization, "-" indicates multiple chromosomal locations for a single gene; "Un" indicates unknown. 


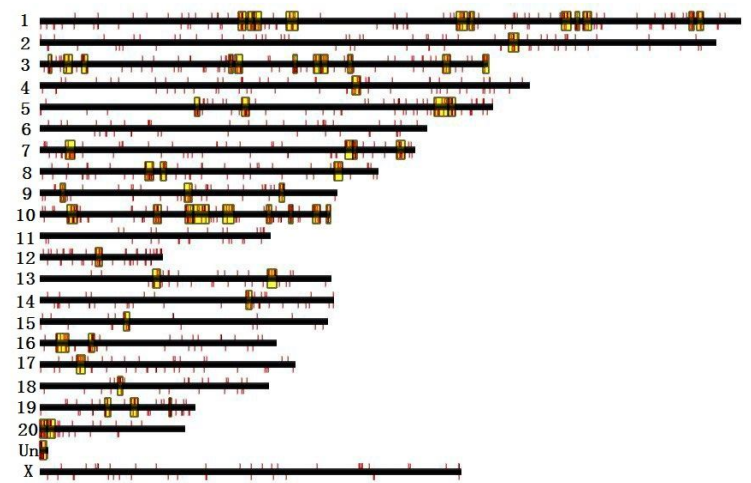

Fig 2: Comparison of chromosomal localization of genes in the wild-type, rat model, and treatment groups (ANOVA analysis, $p<0.05$ ). Note: Red colour indicates individual genes, yellow partially indicates gene-rich regions, and "un" indicates unknown genes that were not localized.

\section{DISCUSSION}

Traditional Chinese medicine (TCM) considers the organic defects of type 2 diabetes to be located mainly in the stomach, liver, and kidneys with some involvement from the cardiopulmonary system. The basic theory of TCM includes fivezang organs and six fuorgans, vital energy (Qi), blood, and meridians (Jingluo). TCM drug treatment typically consists of a complex prescription of multiple components based on differentiation of symptoms and signs (zheng), including Yin, Yang, exterior (biao), interior (li), cold (han), heat (re), deficiency (xu), and excess (shi). Diabetes is categorized by TCM as a mixed disorder involving weakness conditions and presenting conditions in multiple areas. The two main weakness conditions involve Qi or both Qi and Yin, while the presenting conditions involve inner heat, stasis, and turbidity. Previous research indicates that Dendrobium mixtures can improve Yin, soothe inner heat, and replenish the kidney [7]. It has also been found to significantly improve lung and kidney function in STZ rat model. DEN 1 improved Yin and Qi, enhanced circulation, strengthened the spleen, replenishes the kidneys, and reduces inner heat. DEN 2 strengthened life serum, reduced inner heat, and prevented excessive heating. DEN 3 nourishes Yin, reduced humidity and turbidity, and expelled humid heat through the beneficial effect on phlegm and inner heat. We tested the clinically effective drugs in diabetic rat models and treatment group with wild-type group as well as between treated and untreated model groups. In this way, target genes can be identified accurately. Corrected genes were mainly related to metabolic processes, immune processes, cell growth, apoptosis, signal transduction, carbohydrate synthesis, carbohydrate degradation, transcription regulation, olfactory receptors, the microtubule system, and the nervous system.

The CD gene family and toll-like receptor-9 protein (TLR9) were affected by the treatment. Both human and rodent TLR9 have $77 \%$ homology. TLR9 signal transduction ultimately activates transcription factors such as NF2KB, AP21 and CREB, and these transcription factors increase expression of various cytokines and chemokine, thereby promoting the proliferation of $B$ cells and activating macrophages. In this way, it plays an important innate immune defense against pathogens [8,9]. Migita et al used flow cytometry to determine that B lymphocyte TLR9 is up-regulated in autoimmune diseases (systemic lupus erythematosus and autoimmune hemolytic anemia) [10]. Christensen et al have shown that TLR9-deficient mice produce increasing amounts of IFNa and show enhanced immune activation, increased Lupus nephritis, and increased mortality [11]. Our study found that TLR9 receptors in diabetic rats had low expression but returned to normal levels after treatment. This indicates that Dendrobium mixture (DEN) can improve autoimmune activity in diabetic rats, thereby enhancing the ability of the body to counter external pathogens and stimuli.

Our study also found a variety of receptors in the $C D$ (cluster of differentiation) gene family with increased levels of expression in the liver of diabetic rats, such as CD24 ( $F C=3.16), C D 38$ $(F C=1.30), C D 74(F C=1.55), C D 44(F C=$ 1.97). The expression level normalized after Dendrobium mixture treatment, compared with normal group. CD molecules are widely involved in immune cell recognition of antigens, act as receptors for certain immune-active substances, mediate adhesion between cells and matrix, mediate immune response, and are involved in inflammation, hematopoietic regulation, wound healing, blood clotting, tumor metastasis, and other important physiological and pathological processes[12-15]. CD24 may participate in tumor development by promoting tumor cell proliferation, invasion, and metastasis. It has become a prognostic sign, and indicator of invasion and metastasis for certain malignant tumors [12].

CD38 molecule is an indicator for chronic B lymphocytic leukemia, a diagnostic criterion for reactive autoimmune diabetes. It can be used for the detection of AIDS and cytomegalovirus, and to monitor the condition of patients with systemic lupus erythematosus [13]. CD74 is a cell membrane receptor of macrophage migration inhibitory factor (MIF). It has been found to be over-expressed in a variety of cancer tissues 
[14]. Abnormal expression of CD44 is considered to be a biological marker in tumors, early stages of cancer, and cancer metastasis [15]. Overexpression of CD proteins in diabetic patients provides a fertile environment for tumor growth and differentiation. Dendrobium mixture therapy not only changed gene expression patterns in type 2 diabetes but also improved immune activity and reduced the likelihood of cancer development.

Genes that are down-regulated in rats in vivo include L-leucine hydrolase, amine oxidase, lowdensity lipoprotein receptor-related protein 5 , and ATP enzyme (Atp6nl). ATP enzyme is upregulated in rats considered systemically cool by TCM and down-regulated in rats considered systemically warm by TCM [16]. In our study, the ATP enzyme Atp13a1 protein is down-regulated (0.3939) and displays a possible TCM association with extreme heat due to deficiency of Yin, indicating that Dendrobium mixtures can regulate agitated inner heat. Also overexpressed in rats are $G$ proteins and their regulatory proteins, cytochrome $\mathrm{P} 450$, glutathione peroxidase, O-acyltransferase membranebinding domain, lipoprotein lipase, cytidine deaminase, dimethyl amino hydrolase, protein tyrosine acid phosphatase, and Tspan8 protein.

In 2008, more than 40 United States and European Institutes conducted genetic analyses of 70,000 diabetes patients and found six novel genes related to type 2 diabetes. These can be used as molecular markers of diabetes. One of those six genes was Tspan8 [17]. In our experiment, a three-fold increase in Tspan8 expression was observed in the diabetic rat group. This is consistent with previous studies [17]. Normal expression of Tspan8 was restored after drug treatment, confirming this gene as one of the target sites for Dendrobium mixture treatment.

Glutathione peroxidase 2 (Gpx2) is an important antioxidant enzyme which can remove excess peroxides, lipids, and phospholipid-free radicals, and can protect cell membranes and DNA [18]. Gpx2 was found in the present study to be overexpressed in diabetic rats (5.4068 fold). Gamma glutamyl transferase (GGT1) is one of the most important indicators of liver function. Excessive consumption of protein supplements can increase liver burden, leading to elevated GGT [19]. This gene has 4.55-fold higher expression in model rats, which is consistent with the results of other clinical studies. The expression of GGT normalized after treatment, indicating that Dendrobium mixture is hepatoprotective.
WNT1 signaling pathway protein 2 (Wisp2) has been proven to play a key role in a number of cancers. Glypican 3 (Gpc3) has also been implicated in a variety of cancers such as ovarian and liver cancers. Deleted in malignant brain tumors 1 protein (DMBT1) is also associated with a variety of cancers. Heat shock protein beta1 is associated with cancer cell metastasis and photo-oxidized aging of the skin. Keratin has been confirmed to be associated with colorectal carcinoma. Fibulin-like extracellular matrix protein 1 (fibulin1) and Fxyd3 protein have been confirmed as overexpressed in gastric adenocarcinoma, colorectal cancer, pancreatic cancer, and other tissue cancers [20, 21]. A number of apoptosis- and cancer-related genes showed increased expressions in the liver of rats, indicating that the Dendrobium mixture helped to maintain normal cell differentiation and apoptosis.

\section{CONCLUSION}

TCM herbal remedies are complex in composition and not all of the resulting molecular effects on different organisms have been elucidated. Further study into the genetic polymorphisms and validation of protein function of our screened divergent genes will help researchers identify new targets for TCM treatment of type 2 diabetes and lay a firm foundation for molecular diagnosis of clinical diabetes and integrated TCM-Western medical treatment of diabetes

\section{REFERENCES}

1. Zimmet $P$, Alberti KG, Show J. Global and societal implications of the diabetes epidemic[J]. Nature 2001; 414(13): 782-787.

2. Eyre $H$, Kahn R, Robertson RM, Clark NG, Doyle C, Hong $Y$, Gansler T, Glynn T, Smith RA, Taubert $K$ et a1. Preventing cancer, cardiovascular disease, and diabetes: a common agenda for the American Cancer Society, the American Diabetes Association and the American Heart Association [J]. Circulation 2004; 109(25): 3244-3248.

3. Koya $D$, Hayashi K, Kitada M, Kashiwagi A, Kikkawa Ryuichi, Haneda M. Effects of antioxidants in diabetes-induced oxidative stress in glomeruli of diabetic rats[J]. J Am Soc, Nephrol 2003; 14(suppl 3): 250-253

4. Sehulze A, Downwartl J. Navigating gene expression using microarrays a technology review Nat Cell Biol[J]. 2001; 3(8): 190-195

5. Ezziane Z. Applications of artificial intelligence in bioinformatics: a review [J]. Expert Systems with Applications 2006; 30(1): 2-10.

6. Derrell C. Guide for the care and use of laboratory animals. Institute of Laboratory Animal Resources. National Academy Press, Washington DC, USA 1996.

7. Zheng YF, Shi $H$, Yang XZ. Influence on islet cell secretion by dendrobium compound. Chi J Hos Pharm 2010; 30: 551-555. 
8. Stacey KJ, Sweet MJ, Hume DA. Macrophages ingest and are activated by bacterial DNA. J Immunol 1996; 157: 2116-2122.

9. Sparwasser T, Koch ES, Vabulas RM, Heeg K, Lipford GB, Ellwart JW, Wagner H. Bacterial DNA and immunostimulatory CpG oligonucleotides trigger maturation and activation of murine dendritic cells. Eur J Immunol 1998; 28: 2045-2054.

10. Migita $K$, Miyashita $T$, Maeda $Y$, Nakamura $M$, Yatsuhashi $H$, Kimura $H$, Ishibashi $H$, Eguchi $K$. Toll-like receptor expression in lupus peripheral blood mononuclear cells. J Rheumatol 2007; 34: 493-500.

11. Christensen SR, Shupe J, Nickerson K, Kashgarian M, Flavell RA, Shlomchik MJ. Toll-like receptor 7 and TLR9 dictate autoantibody specificity and have opposing inflammatory and regulatory roles in a murine model of lupus. Immunity 2006; 25: 417 428.

12. Kristiansen G, Winzer $K J$, Mayordomo E, Bellach J, Schlüns K, Denkert C, Dahl E, et al. CD24 expression is a new prognostic marker in breast cancer. Clin Cancer Res 2003; 9: 4906-4913.

13. Deshpande DA, White TA, Dogan S, Walseth TF, Panettieri RA, Kannan MS. CD38/cyclic ADPribose signaling:role in the regulation of calcium homeostasis in airway smooth muscle. $A m \mathrm{~J}$ Physiol Lung Cell Mol Physiol 2005; 288: 773-788

14. Lian JF, Wu SH, Shang HX, Zhang Li-li, Gao YR Expression of MIF and receptor CD74 and their significance in cervical cancer. Chi Remedies Clinics 2011; 7: 755-757
15. Qian Hao, Ling PX, Wang FS, Zhang TM. Research progress in the relationship between CD44 and malignant tumor. Chi J Biochem Pharmaceut 2007; 3: 207-210.

16. Wei BB, Zhang WF, Zhang RY, Wang XL, Huang JF, Zhang WR. Analysis of RT-PCR on Difference of Gene Expression in Rats of Cold Body and Heat Body Constitution. Acta Univer Tradit Med Sin Pharm Shanghai 2011; 3: 68-70.

17. Zeggini E, Scott LJ, Saxena R, Voight BF, Marchini JL, Hu T, de Bakker PIW et al. Meta-analysis of genome-wide association data and large-scale replication identifies additional susceptibility loci for type 2 diabetes. Nat Genet 2008; 40: 638-645.

18. Zhao H, Zhou JC, Li JG, Zhao Y, Wang KN. Study on the cloning and isolation of sus scrofa GPX2 Gene by RACE Method. Agric Sci and Technol 2008; 36: 7586-7588.

19. Cao JM. Relation of serum y - glutamyltransferase to obesity and insulin resistance. Chi J Med Lab Technol 2010;11: 70-72.

20. Kayed H, Kleeff J, Kolb A, Ketterer K, Keleg S, Felix K, Giese T, Penzel R, Zentgraf $H$, Büchler MW, et al. FXYD3 is overexpressed in pancreatic ductal adenocarcinoma and influences pancreatic cancer cell growth. Int J Cancer 2006; 118: 43-54

21. Yamamoto $H$, Okumura $K$, Toshima $S$, Mukaisho $K$, Sugihara $H$, Hattori $T$, Kato M, Asano S. FXYD3 protein involved in tumor cell proliferation is overproduced in human breast cancer tissues. Biol Pharm Bull 2009; 32: 1148-1154. 